\title{
Posets from Admissible Coxeter Sequences
}

\author{
Matthew Macauley \\ Department of Mathematical Sciences \\ Clemson University \\ Clemson, South Carolina, USA \\ macaule@clemson.edu
}

\author{
Henning S. Mortveit \\ Department of Mathematics \\ Virginia Bioinformatics Institute \\ Virginia Tech \\ Blacksburg, Virginia, USA \\ henning.mortveit@vt.edu
}

Submitted: Apr 6, 2010; Accepted: Sep 24, 2011; Published: Oct 3, 2011

Mathematics Subject Classification: 20F55;06A06;05C20

\begin{abstract}
We study the equivalence relation on the set of acyclic orientations of an undirected graph $\Gamma$ generated by source-to-sink conversions. These conversions arise in the contexts of admissible sequences in Coxeter theory, quiver representations, and asynchronous graph dynamical systems. To each equivalence class we associate a poset, characterize combinatorial properties of these posets, and in turn, the admissible sequences. This allows us to construct an explicit bijection from the equivalence classes over $\Gamma$ to those over $\Gamma^{\prime}$ and $\Gamma^{\prime \prime}$, the graphs obtained from $\Gamma$ by edge deletion and edge contraction of a fixed cycle-edge, respectively. This bijection yields quick and elegant proofs of two non-trivial results: $(i)$ A complete combinatorial invariant of the equivalence classes, and $(i i)$ a solution to the conjugacy problem of Coxeter elements for simply-laced Coxeter groups. The latter was recently proven by H. Eriksson and K. Eriksson using a much different approach.
\end{abstract}

\section{Overview}

Let $O_{\Gamma}$ be an acyclic orientation of the undirected graph $\Gamma$. A cyclic 1-shift (left) of a linear extension $\pi$ of $O_{\Gamma}$ corresponds to converting a source (the element $\pi_{1}$ ) of $O_{\Gamma}$ into a sink, and this gives rise to an equivalence relation on $\operatorname{Acyc}(\Gamma)$ denoted by $\sim_{\kappa}$. We let $\kappa(\Gamma)$ denote the number of equivalence classes in $\operatorname{Acyc}(\Gamma)$ under $\sim_{\kappa}$, and refer to the equivalence classes as $\kappa$-classes.

This paper is organized as follows. After terminology and background in Section 2, we show in Section 3 how to associate a poset to each $\kappa$-class, and we characterize structural properties of these posets. This helps us better understand admissible sequences as a whole, culminating in a bijection

$$
\Theta: \operatorname{Acyc}(\Gamma) / \sim_{\kappa} \longrightarrow\left(\operatorname{Acyc}\left(\Gamma_{e}^{\prime}\right) / \sim_{\kappa}\right) \bigcup\left(\operatorname{Acyc}\left(\Gamma_{e}^{\prime \prime}\right) / \sim_{\kappa}\right)
$$


where $\Gamma_{e}^{\prime}$ and $\Gamma_{e}^{\prime \prime}$ are the graphs formed by deleting and contracting a cycle-edge $e$ of $\Gamma$, respectively. From this bijection, the recursion relation for $\kappa(\Gamma)$ in $[10]$ becomes an immediate corollary, enumerating $\kappa(\Gamma)$ through an evaluation of the Tutte polynomial. In Section 4 , we use our bijection to construct a complete invariant of $\operatorname{Acyc}(\Gamma) / \sim_{\kappa}$, the set of $\kappa$-classes of $\Gamma$. In Section 5, we review a connection to Coxeter theory, and show how the prior results easily solve the conjugacy problem for Coxeter elements in all simply-laced Coxeter groups, and how $\kappa(\Gamma)$ enumerates the conjugacy classes of Coxeter elements. Finally, in the summary, we briefly discuss how the equivalence relation $\sim_{\kappa}$ arises in other areas of mathematics such as sequential dynamical systems, the chip-firing game, and the representation theory of quivers. Throughout the paper, we maintain a running example (that we visit five times) using a six-vertex graph $\Gamma$ that should enhance the paper's readability and motivate the main ideas.

\section{Terminology and Background}

Let $\Gamma$ be an undirected, simple and loop-free graph with vertex set $\mathrm{v}[\Gamma]=\{1,2, \ldots, n\}$ and edge set e $[\Gamma]$. Let $S_{\Gamma}$ denote the set of total orders (i.e., permutations) of $\mathrm{v}[\Gamma]$. Define a relation $\sim$ on $S_{\Gamma}$ where $\pi \sim \pi^{\prime}$ if $\pi=\pi_{1} \pi_{2} \cdots \pi_{n}$ and $\pi^{\prime}=\pi_{1}^{\prime} \pi_{2}^{\prime} \cdots \pi_{n}^{\prime}$ differ by a single adjacent transposition $\pi_{i} \pi_{i+1} \mapsto \pi_{i+1} \pi_{i}$ where $\left\{\pi_{i}, \pi_{i+1}\right\} \notin \mathrm{e}[\Gamma]$. The reflexive transitive closure of $\sim$ is an equivalence relation on $S_{\Gamma}$ denoted by $\sim_{\alpha}$. We denote the equivalence class containing $\pi$ by $[\pi]_{\Gamma}$, and set

$$
S_{\Gamma} / \sim_{\alpha}=\left\{[\pi]_{\Gamma} \mid \pi \in S_{\Gamma}\right\}
$$

This corresponds to partially commutative monoids as defined in [4], but restricted to fixed length permutations over $\mathrm{v}[\Gamma]$ and with commutation relations encoded by non-adjacency in the graph $\Gamma$. Those familiar with Coxeter theory will recognize the similarity of these equivalence classes and the commutation classes [20] of reduced expressions of Coxeter elements.

Orientations of $\Gamma$ are represented as maps $O_{\Gamma}: \mathrm{e}[\Gamma] \longrightarrow \mathrm{v}[\Gamma] \times \mathrm{v}[\Gamma]$, which may also be viewed as directed graphs. The set of acyclic orientations of $\Gamma$ is denoted by $\operatorname{Acyc}(\Gamma)$, and we set $\alpha(\Gamma)=|\operatorname{Acyc}(\Gamma)|$. Each acyclic orientation defines a partial ordering on $\mathrm{v}[\Gamma]$ where the covering relations are $i \leq_{O_{\Gamma}} j$ if $\{i, j\} \in \mathrm{e}[\Gamma]$ and $O_{\Gamma}(\{i, j\})=(i, j)$. The set of linear extensions of $O_{\Gamma}$ contains precisely the permutations $\pi \in S_{\Gamma}$ such that if $i \leq_{O_{\Gamma}} j$, then $i$ precedes $j$ in $\pi$. Through the ordering of $\mathrm{v}[\Gamma]$, every permutation $\pi \in S_{\Gamma}$ induces a canonical linear order on $\mathrm{v}[\Gamma]$. Moreover, each permutation $\pi \in S_{\Gamma}$ induces an acyclic orientation $O_{\Gamma}^{\pi} \in \operatorname{Acyc}(\Gamma)$ defined by $O_{\Gamma}^{\pi}(\{i, j\})=(i, j)$ if $i$ precedes $j$ in $\pi$ and $O_{\Gamma}^{\pi}(\{i, j\})=(j, i)$ otherwise. The canonical bijection

$$
f_{\Gamma}: S_{\Gamma} / \sim_{\alpha} \longrightarrow \operatorname{Acyc}(\Gamma), \quad f_{\Gamma}\left([\pi]_{\Gamma}\right)=O_{\Gamma}^{\pi}
$$

identifies equivalence classes and acyclic orientations, and thus the number of equivalence classes under $\sim_{\alpha}$ is $\alpha(\Gamma)$. 
For $O_{\Gamma} \in \operatorname{Acyc}(\Gamma)$ and $e=\{v, w\} \in \mathrm{e}[\Gamma]$, let $O_{\Gamma}^{\rho(e)}$ be the orientation of $\Gamma$ obtained from $O_{\Gamma}$ by reversing the orientation of the edge $e$. Let $\Gamma_{e}^{\prime}$ and $\Gamma_{e}^{\prime \prime}$ denote the graphs obtained from $\Gamma$ by deletion and contraction (see, e.g., [13, p. 415]) of $e$, respectively, and let $O_{\Gamma^{\prime}}$ and $O_{\Gamma^{\prime \prime}}$ denote the orientations of $O_{\Gamma}$ inherited under these operations. (Since our graphs are assumed to be loop-free, when we contract an edge $\{v, w\}$, we remove the resulting loop.) The bijection

$$
\beta_{e}: \operatorname{Acyc}(\Gamma) \longrightarrow \operatorname{Acyc}\left(\Gamma_{e}^{\prime}\right) \cup \operatorname{Acyc}\left(\Gamma_{e}^{\prime \prime}\right)
$$

defined by

$$
O_{\Gamma} \stackrel{\beta_{e}}{\longmapsto} \begin{cases}O_{\Gamma^{\prime}}, & O_{\Gamma}^{\rho(e)} \notin \operatorname{Acyc}(\Gamma), \\ O_{\Gamma^{\prime}}, & O_{\Gamma}^{\rho(e)} \in \operatorname{Acyc}(\Gamma) \text { and } O_{\Gamma}(e)=(v, w), \\ O_{\Gamma^{\prime \prime}}, & O_{\Gamma}^{\rho(e)} \in \operatorname{Acyc}(\Gamma) \text { and } O_{\Gamma}(e)=(w, v),\end{cases}
$$

is well-known, and shows that one may compute $\alpha(\Gamma)$ through the recursion relation

$$
\alpha(\Gamma)=\alpha\left(\Gamma_{e}^{\prime}\right)+\alpha\left(\Gamma_{e}^{\prime \prime}\right),
$$

valid for any $e \in \mathrm{e}[\Gamma]$. It basically removes the edge $e=\{v, w\}$ if it cannot be contracted, and otherwise, it either contracts or removes it depending on its orientation. We illustrate this with the following example, which we will revisit four more times throughout this article.

Example 2.1. For an explicit example of $\beta_{e}$, see Figure 1, which shows three acyclic orientations of the same graph $\Gamma$, and a fixed edge $e=\{v, w\}$. (The vertices $v^{\prime}, w^{\prime}$, and $z$, which will be referred to later, are only labeled once for clarity.) Call these orientations $O_{\Gamma}^{a}, O_{\Gamma}^{b}$, and $O_{\Gamma}^{c}$, respectively. The map $\beta_{e}$ removes edge e from $O_{\Gamma}^{a}$ because contracting it would result in a directed cycle. Neither $O_{\Gamma}^{b}$ nor $O_{\Gamma}^{c}$ have a directed path from $v$ to $w$ other than the edge $(v, w)$, so in both cases, contracting e would give an acyclic orientation of $\Gamma_{e}^{\prime \prime}$. By the definition of $\beta_{e}$ in (4), $\beta_{e}$ removes e in $O_{\Gamma}^{b}$, and contracts it in $O_{\Gamma}^{c}$.

Via the bijection in (2), it is clear that mapping $\pi=\pi_{1} \pi_{2} \cdots \pi_{n} \in[\pi]_{\Gamma}$ to $\pi_{2} \cdots \pi_{n} \pi_{1}$ corresponds precisely to converting the source vertex $\pi_{1}$ in $O_{\Gamma}^{\pi}$ into a sink. We call such a conversion a source-to-sink operation, or a click. Two orientations $O_{\Gamma}, O_{\Gamma}^{\prime} \in \operatorname{Acyc}(\Gamma)$ where $O_{\Gamma}$ can be transformed into $O_{\Gamma}^{\prime}$ by a sequence of clicks are said to be click-related. We write this as $\mathbf{c}\left(O_{\Gamma}\right)=O_{\Gamma}^{\prime}$ where $\mathbf{c}=c_{1} c_{2} \cdots c_{k}$ with $c_{i} \in \mathrm{v}[\Gamma]$. To clarify notation, we mean

$$
\mathbf{c}\left(O_{\Gamma}\right)=c_{k}\left(c_{k-1}\left(\cdots c_{2}\left(c_{1}\left(O_{\Gamma}\right)\right)\right)\right) .
$$

Such as sequence $\mathbf{c}$ is called an admissible sequence, or a click-sequence. The former term comes from the representation theory of quivers $[1,8]$, but we will usually stick to the latter due to brevity, the overuse of the term "admissible sequence" throughout mathematics, and the convenience of "click" doubling as a verb. It is straightforward to verify that this click-relation is an equivalence relation on $\operatorname{Acyc}(\Gamma)$, and we also refer to click-related 


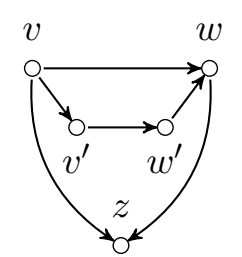

(a) $O_{\Gamma}^{a}$

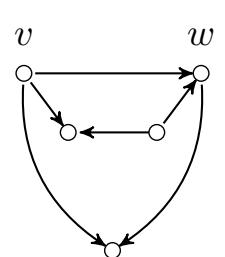

(b) $O_{\Gamma}^{b}$

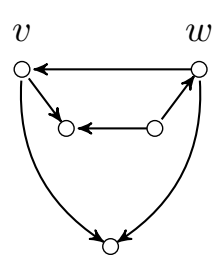

(c) $O_{\Gamma}^{c}$

Figure 1: An example of the map $\beta_{e}$ applied to three acyclic orientations of a graph $\Gamma$. If contracting the edge $e=\{v, w\}$ would introduce a directed cycle (as in $O_{\Gamma}^{a}$ ), then we must delete it. Otherwise, we can either delete or contract it, so we pick the convention that we delete it if it is oriented $(v, w)$ (as in $O_{\Gamma}^{b}$ ), and contract it if is oriented $(w, v)$ (as in $O_{\Gamma}^{c}$ ).

acyclic orientations as $\kappa$-equivalent. Clearly, and as pointed out by V. Reiner [15, p. 309], one may also approach this in the setting of total orders on $\mathrm{v}[\Gamma]$ by identifying elements that differ by $(i)$ flips of adjacent elements not connected in $\Gamma$ and $(i i)$ cyclic shifts. However, for our purposes, approaching this at the level of acyclic orientations seems more natural in light of the bijection (2).

\section{Constructing the Bijection $\Theta$}

The bijection $\beta_{e}: \operatorname{Acyc}(\Gamma) \longrightarrow \operatorname{Acyc}\left(\Gamma_{e}^{\prime}\right) \cup \operatorname{Acyc}\left(\Gamma_{e}^{\prime \prime}\right)$ in $(3)$ does not extend to a welldefined map on $\kappa$-classes, i.e., $\operatorname{Acyc}(\Gamma) / \sim_{\kappa} \longrightarrow\left(\operatorname{Acyc}\left(\Gamma_{e}^{\prime}\right) / \sim_{\kappa}\right) \cup\left(\operatorname{Acyc}\left(\Gamma_{e}^{\prime \prime}\right) / \sim_{\kappa}\right)$. Thus, we need to take a different approach to construct our bijection. An edge $e$ of an undirected graph $\Gamma$ is a bridge if removing $e$ increases the number of connected components of $\Gamma$. An edge that is not a bridge is a cycle-edge, or equivalently, an edge $e$ is a cycle-edge if it is contained in a cycle traversing $e$ precisely once.

Throughout, we will let $e=\{v, w\}$ denote a fixed cycle-edge of the connected graph $\Gamma$, and, for ease of notation, we set $\Gamma^{\prime}=\Gamma_{e}^{\prime}$ and $\Gamma^{\prime \prime}=\Gamma_{e}^{\prime \prime}$. Recall that for $O_{\Gamma} \in \operatorname{Acyc}(\Gamma)$ we let $O_{\Gamma^{\prime}}$ and $O_{\Gamma^{\prime \prime}}$ denote the inherited orientations of $\Gamma^{\prime}$ and $\Gamma^{\prime \prime}$. Notice that $O_{\Gamma^{\prime}}$ is always acyclic, while $O_{\Gamma^{\prime \prime}}$ is acyclic if and only if there is no directed path with endpoints $v$ and $w$ in $O_{\Gamma^{\prime}}$. Finally, we let $\left[O_{\Gamma}\right]$ denote the $\kappa$-class containing $O_{\Gamma}$.

The interval $[a, b]$ of a poset $\mathcal{P}$ (where $a \leq b$ ) is the subposet consisting of all $c \in \mathcal{P}$ such that $a \leq c \leq b$. Viewing a finite poset $\mathcal{P}$ as a directed graph $D_{\mathcal{P}}$, the interval $[a, b]$ contains precisely the vertices that lie on a directed path from $a$ to $b$, and thus is a vertexinduced subgraph of $D_{\mathcal{P}}$. By assumption, e[Г] contains $\{v, w\}$, so for all $O_{\Gamma} \in \operatorname{Acyc}(\Gamma)$ either $v \leq_{O_{\Gamma}} w$ or $w \leq_{O_{\Gamma}} v$. In this section, we will study the interval $[v, w]$ in the poset $O_{\Gamma}$ (when $\left.v \leq_{O_{\Gamma}} w\right)$ and its behavior under clicks.

Definition 3.1. Let $\mathrm{Acyc}_{\leq}(\Gamma)$ be the set of acyclic orientations of vertex-induced subgraphs of $\Gamma$. Define the map

$$
\mathcal{I}: \operatorname{Acyc}(\Gamma) \longrightarrow \operatorname{Acyc}_{\leq}(\Gamma)
$$


by $\mathcal{I}\left(O_{\Gamma}\right)=[v, w]$ if $v \leq_{O_{\Gamma}} w$, and by $\mathcal{I}\left(O_{\Gamma}\right)=\varnothing$ (the null graph) otherwise. We will refer to $\mathcal{I}\left(O_{\Gamma}\right)$ as the vw-interval of $O_{\Gamma}$.

Elements of $\operatorname{Acyc}(\Gamma)$ can be thought of as posets over $\mathrm{v}[\Gamma]$, and elements of $\operatorname{Acyc}_{\leq}(\Gamma)$ can be thought of as certain subposets of these, though they need not be induced (because two vertices on a directed path in $\Gamma$ need not be on a directed path in an induced subgraph of $\Gamma)$. Through a slight abuse of notation, we will at times refer to $\mathcal{I}\left(O_{\Gamma}\right)$ as a poset, a directed graph, or a subset of $\mathrm{v}\left[O_{\Gamma}\right]$. In this last case, it is understood that the relations are inherited from $O_{\Gamma}$.

Let $P$ be an undirected path in $\Gamma$ of length- $k$, i.e., $P=v_{0}, v_{1}, \ldots, v_{k-1}, v_{k}$ where $\left\{v_{i-1}, v_{i}\right\} \in \mathrm{e}[\Gamma]$ for $i=1, \ldots, k$. Define the function

$$
\nu_{P}: \operatorname{Acyc}(\Gamma) \longrightarrow \mathbb{Z},
$$

where $\nu_{P}\left(O_{\Gamma}\right)$ is the number of edges in $\Gamma$ of the form $\left\{v_{i-1}, v_{i}\right\}$ oriented as $\left(v_{i-1}, v_{i}\right)$ in $O_{\Gamma}$, minus the number of edges oriented as $\left(v_{i}, v_{i-1}\right)$. If $P$ is a cycle (i.e., $\left.v_{0}=v_{k}\right), \nu_{P}$ is preserved under clicks, and thus in this case, it extends to a map $\nu_{P}^{*}: \operatorname{Acyc}(\Gamma) / \sim_{\kappa} \longrightarrow \mathbb{Z}$. In [18], J.-Y. Shi defines this function for Coxeter graphs containing a single cycle, referring to it as Coleman's $\nu$-function (see [5]). The definition given here is more general, and will allow us to extend Shi's characterization of conjugacy classes to include all simply-laced Coxeter groups.

Example 3.2. Continuing with Example 2.1, consider the three orientations in Figure 1, whose vw-intervals are the following:

$$
\mathcal{I}\left(O_{\Gamma}^{a}\right)=\left\{v, w, v^{\prime}, w^{\prime}\right\}, \quad \mathcal{I}\left(O_{\Gamma}^{b}\right)=\{v, w\}, \quad \mathcal{I}\left(O_{\Gamma}^{c}\right)=\varnothing .
$$

Next, consider the undirected (but oriented) path $P=v, v^{\prime}, w^{\prime}, w, v$ (a cycle) and the corresponding map $\nu_{P}$, as defined in (5). It is easy to check that

$$
\nu_{P}\left(O_{\Gamma}^{a}\right)=2, \quad \nu_{P}\left(O_{\Gamma}^{b}\right)=0, \quad \nu_{P}\left(O_{\Gamma}^{c}\right)=2 .
$$

We conclude that $O_{\Gamma}^{b}$ cannot be $\kappa$-equivalent to either $O_{\Gamma}^{a}$ or $O_{\Gamma}^{c}$. Finally, if we consider the undirected path $Q=v, z, w, v$, we have

$$
\nu_{Q}\left(O_{\Gamma}^{a}\right)=-1, \quad \nu_{Q}\left(O_{\Gamma}^{b}\right)=-1, \quad \nu_{Q}\left(O_{\Gamma}^{c}\right)=1 .
$$

Therefore, $O_{\Gamma}^{a} \nsim_{\kappa} O_{\Gamma}^{c}$, and hence all three of these orientations lie in distinct $\kappa$-classes.

As we will see in Section 4, when taken over all cycles of $\Gamma$, the $\nu$-function is a actually a complete invariant, i.e., $O \sim_{\kappa} O^{\prime}$ if and only iff $\nu_{C}(O)=\nu_{C}\left(O^{\prime}\right)$ for all cycles $C$ in $\Gamma$. First, we need to establish a series of structural results about the $v w$-interval. Since $\{v, w\} \in \mathrm{e}[\Gamma]$, every $\kappa$-class contains at least one orientation $O_{\Gamma}$ with $v \leq_{O_{\Gamma}} w$, and thus there is at least one element $O_{\Gamma}$ in each $\kappa$-class with $\mathcal{I}\left(O_{\Gamma}\right) \neq \varnothing$. As the next result shows, this (non-empty) choice of $v w$-interval is independent of the choice of representative from $\left[O_{\Gamma}\right]$, meaning that there is a well-defined notion of the $v w$-interval of a $\kappa$-equivalence class. We formalize this by extending the map $\mathcal{I}: \operatorname{Acyc}(\Gamma) \rightarrow \operatorname{Acyc}_{\leq}(\Gamma)$ to a map $\mathcal{I}^{*}: \operatorname{Acyc}(\Gamma) / \sim_{\kappa} \rightarrow \operatorname{Acyc}_{\leq}(\Gamma)$. 
Proposition 3.3. The map $\mathcal{I}$ can be extended to a map

$$
\mathcal{I}^{*}: \operatorname{Acyc}(\Gamma) / \sim_{\kappa} \longrightarrow \operatorname{Acyc}_{\leq}(\Gamma) \text { by } \mathcal{I}^{*}\left(\left[O_{\Gamma}\right]\right)=\mathcal{I}\left(O_{\Gamma}^{1}\right),
$$

where $O_{\Gamma}^{1}$ is any element of $\left[O_{\Gamma}\right]$ for which $\mathcal{I}\left(O_{\Gamma}^{1}\right) \neq \varnothing$.

Proof. It suffices to prove that $\mathcal{I}^{*}$ is well-defined. Consider $O_{\Gamma}^{1} \sim_{\kappa} O_{\Gamma}^{2}$ with $v \leq_{O_{\Gamma}^{i}} w$ for $i=1$, 2. Clearly, $\mathcal{I}\left(O_{\Gamma}^{1}\right)$ and $\mathcal{I}\left(O_{\Gamma}^{2}\right)$ contain $v$ and $w$, so suppose that $a \in \mathcal{I}\left(O_{\Gamma}^{1}\right) \backslash\{v, w\}$. Then $a$ lies on a directed path $P^{\prime}$ from $v$ to $w$ in $O_{\Gamma}^{1}$, of length $k \geq 2$ (i.e., $P^{\prime}$ traverses at least 2 edges). Let $P$ be the cycle formed by adding vertex $v$ to the end of $P^{\prime}$. Clearly $\nu_{P}\left(O_{\Gamma}^{1}\right)=k-1$ since $O_{\Gamma}^{1}(e)=(v, w)$.

By assumption, $O_{\Gamma}^{2} \in\left[O_{\Gamma}^{1}\right]$ with $v \leq_{O_{\Gamma}^{2}} w$. Since $\nu_{P}$ is constant on $\left[O_{\Gamma}^{1}\right]$ it follows from $\nu_{P}\left(O_{\Gamma}^{1}\right)=k-1=\nu_{P}\left(O_{\Gamma}^{2}\right)$ that every edge of $P^{\prime}$ is oriented identically in $O_{\Gamma}^{1}$ and $O_{\Gamma}^{2}$, and hence that every directed path $P^{\prime}$ in $O_{\Gamma}^{1}$ is contained in $O_{\Gamma}^{2}$ as well. Therefore, $a \in \mathcal{I}\left(O_{\Gamma}^{2}\right)$, and the reverse inclusion follows by an identical argument.

In light of Proposition 3.3, we define the $v w$-interval of a $\kappa$-class $\left[O_{\Gamma}\right]$ to be $\mathcal{I}^{*}\left(\left[O_{\Gamma}\right]\right)$. The $v w$-interval will be central in understanding properties of click-sequences. First, we make a simple observation without proof; it also appears in [19] in the context of admissible sequences in Coxeter theory.

Proposition 3.4. Let $O_{\Gamma} \in \operatorname{Acyc}(\Gamma)$, let $\mathbf{c}=c_{1} c_{2} \cdots c_{m}$ be an associated click-sequence, and consider any directed edge $\left(v_{1}, v_{2}\right)$ in $O_{\Gamma}$. Then the occurrences of $v_{1}$ and $v_{2}$ in $\mathbf{c}$ alternate, with $v_{1}$ appearing first.

Because $\{v, w\} \in \mathrm{e}[\Gamma]$, we can say more about the vertices in $\mathcal{I}\left(O_{\Gamma}\right)$ that appear between successive instances of in $v$ and $w$ in a click-sequence.

Proposition 3.5. Let $O_{\Gamma} \in \operatorname{Acyc}(\Gamma)$, and let $\mathbf{c}=c_{1} c_{2} \cdots c_{m}$ be an associated clicksequence that contains every vertex of $\mathcal{I}\left(O_{\Gamma}\right)$ at least once and with $c_{1}=v$. Then every vertex of $\mathcal{I}\left(O_{\Gamma}\right)$ appears in $\mathbf{c}$ before any vertex in $\mathcal{I}\left(O_{\Gamma}\right)$ appears twice.

Proof. The proof is by contradiction. Assume the statement is false, and let $a \in \mathcal{I}\left(O_{\Gamma}\right)$ be the first vertex whose second instance in $\mathbf{c}$ occurs before the first instance of some other vertex $z \in \mathcal{I}\left(O_{\Gamma}\right)$. If $a \neq v$, then $a$ is not a source in $O_{\Gamma}$, and there exists a directed edge $\left(a^{\prime}, a\right)$. By Proposition 3.4, $a^{\prime}$ must appear in $\mathbf{c}$ before the first instance of $a$, but also between the two first instances of $a$. This is impossible, because $a$ was chosen to be the first vertex appearing twice in $\mathbf{c}$. That only leaves $a=v$, and $v$ must appear twice before the first instance of $w$. However, this contradicts the statement of Proposition 3.4 because $\{v, w\} \in \mathrm{e}[\Gamma]$.

The next result shows that for any click-sequence $\mathbf{c}$ that contains every element in $\mathcal{I}\left(O_{\Gamma}\right)$ precisely once, we may assume without loss of generality that the vertices in $\mathcal{I}\left(O_{\Gamma}\right)$ appear consecutively. 
Proposition 3.6. Let $O_{\Gamma} \in \operatorname{Acyc}(\Gamma)$ be an acyclic orientation with $v \leq_{O_{\Gamma}} w$. If $\mathbf{c}=$ $c_{1} c_{2} \cdots c_{m}$ is an associated click-sequence containing precisely one instance of $w$, and no subsequent instances of vertices from $\mathcal{I}\left(O_{\Gamma}\right)$, then there exists a click-sequence $\mathbf{c}^{\prime}=$ $c_{1}^{\prime} c_{2}^{\prime} \cdots c_{m}^{\prime}$ such that $(i)$ there exists an interval $[p, q]$ of $\mathbb{N}$ with $c_{j}^{\prime} \in \mathcal{I}\left(O_{\Gamma}\right)$ iff $p \leq j \leq q$, and $(i i) \mathbf{c}\left(O_{\Gamma}\right)=\mathbf{c}^{\prime}\left(O_{\Gamma}\right)$.

Proof. We prove the proposition by constructing a desired click-sequence $\mathbf{c}^{\prime \prime}$ from $\mathbf{c}$ through a series of transpositions where each intermediate click-sequence $\mathbf{c}^{\prime}$ satisfies $\mathbf{c}\left(O_{\Gamma}\right)=\mathbf{c}^{\prime}\left(O_{\Gamma}\right)$. Such transpositions are said to have property $T$.

Let $I=\mathcal{I}\left(O_{\Gamma}\right)$, and let $A$ be the set of vertices in $I^{c}=\mathrm{v}[\Gamma] \backslash I$ that lie on a directed path in $O_{\Gamma}$ to a vertex in $I$ (vertices above $I$ ), and let $B$ be the set of vertices that lie on a directed path in $O_{\Gamma}$ from a vertex in $I$ (vertices below $I$ ). Let $C$ be the complement of $I \cup A \cup B$. Two vertices $c_{i}, c_{j} \in A \cup B$ with $i<j$ for which there is no element $c_{k} \in A \cup B$ with $i<k<j$ are said to be tight. We will investigate when transpositions of tight vertices in a click-sequence $\mathbf{c}$ of $O_{\Gamma}$ has property $T$, and we will see that this is always the case if $c_{i} \in B$ and $c_{j} \in A$. Consider the intermediate acyclic orientation after applying successive clicks $c_{1} c_{2} \cdots c_{i-1}$ to $O_{\Gamma}$. Obviously, $c_{i}$ is a source. At this point, if $c_{j}$ were not a source, then there would be an adjacent vertex $a \in A$ with the edge $\left\{a, c_{j}\right\}$ oriented $\left(a, c_{j}\right)$. For $c_{j}$ to be clicked as usual (i.e., as a source), $a$ must be clicked first, but this would break the assumption that $c_{i}$ and $c_{j}$ are tight. Therefore, $c_{i}$ and $c_{j}$ are both sources at this intermediate step, and so the vertices $c_{i}, c_{i+1}, \ldots, c_{j}$ are an independent set of sources, and may be permuted in any manner without changing the image of the click sequence. Therefore, the transposition of $c_{i}$ and $c_{j}$ in $\mathbf{c}$ has property $T$, as claimed. By iteratively transposing tight pairs in $\mathbf{c}$, we can construct a click-sequence with the property that every vertex in $A$ comes before every vertex in $B$. In light of this, we may assume without loss of generality that $\mathbf{c}$ has this property.

The next step is to show that we can move all vertices in $A$ before $v$, and all vertices in $B$ after $w$ via transpositions having property $T$. Let $a$ be the first vertex in $A$ appearing after $v$ in the click sequence $\mathbf{c}$. We claim that the transposition moving $a$ to the position directly preceding $v$ has property $T$. This is immediate from the observation that when $v$ is to be clicked, $a$ is a source as well, by the definition of $A$, thus it may be clicked before $v$, without preventing subsequent clicks of vertices up until the original position of $a$. Therefore, we may one-by-one move the vertices in $A$ that are between $v$ and $w$, in front of $v$. An analogous argument shows that we may move the vertices in $B$ that appear before $w$ to a position directly following $w$. In the resulting click-sequence $\mathbf{c}^{\prime}$, the only vertices between $v$ and $w$ are either in $I$ or $C$. The subgraph of the directed graph $O_{\Gamma}$ induced by $C$ is a disjoint union of weakly connected components, and none of the vertices are adjacent to $I$. By definition of $A$ and $B$, there cannot exist a directed edge $(c, a)$ or $(b, c)$, where $a \in A, b \in B$, and $c \in C$. Thus for each weakly connected component of $C$, the vertices in the component can be moved within $\mathbf{c}^{\prime}$, preserving their relative order, to a position either ( $i$ ) directly after the vertices in $A$ and before $v$, or (ii) directly after $w$ and before the vertices of $B$. Call this resulting click-sequence $\mathbf{c}^{\prime \prime}$. As we just argued, all the transpositions occurring in the rearrangement $\mathbf{c} \mapsto \mathbf{c}^{\prime \prime}$ has property $T$, and $\mathbf{c}^{\prime \prime}$ contains all of the vertices in $I$ in consecutive order, and this proves the result. 
We remark that the last two results together imply that for the interval $[p, q]$ in the statement of Proposition 3.6, $c_{p}=v, c_{q}=w$, and the sequence $c_{p} c_{p+1} \cdots c_{q}$ contains every vertex in $\mathcal{I}\left(O_{\Gamma}\right)$ precisely once. A simple induction argument implies the following.

Corollary 3.7. Suppose that $O_{\Gamma} \in \operatorname{Acyc}(\Gamma)$ with $v \leq_{O_{\Gamma}} w$, and let $\mathbf{c}=c_{1} c_{2} \cdots c_{m}$ be a click-sequence where $w$ appears exactly $k$ times, and no vertex from $\mathcal{I}\left(O_{\Gamma}\right)$ appears in $\mathbf{c}$ after the last instance of $w$. Then there exists a click-sequence $\mathbf{c}^{\prime}=c_{1}^{\prime} c_{2}^{\prime} \cdots c_{m}^{\prime}$ such that $(i)$ there are $k$ disjoint intervals $\left[p_{i}, q_{i}\right]$ of $\mathbb{N}$ such that $c_{j} \in \mathcal{I}\left(O_{\Gamma}\right)$ iff $p_{i} \leq j \leq q_{i}$ for some $i$, and $(i i) \mathbf{c}\left(O_{\Gamma}\right)=\mathbf{c}^{\prime}\left(O_{\Gamma}\right)$.

Proof. The argument is by induction on $k$. When $k=1$, the statement is simply Proposition 3.6. Suppose the statement holds for all $k \leq N$, for some $N \in \mathbb{N}$, and let c be a click-sequence containing $N+1$ instances of $w$. Let $c_{\ell}$ be the second instance of $v$ in $\mathbf{c}$, and consider the two click-sequences $\mathbf{c}_{i}:=c_{1} c_{2} \cdots c_{\ell-1}$ and $\mathbf{c}_{f}:=c_{\ell} c_{\ell+1} \cdots c_{m}$. By Proposition 3.6, there exists an interval $\left[p_{1}, q_{1}\right]$ with $p_{1}<q_{1}<\ell$, and by the induction hypothesis, there exists $k$ intervals $\left[p_{2}, q_{2}\right], \ldots,\left[p_{k+1}, q_{k+1}\right]$ with $\ell \leq p_{2}<q_{2}<\cdots<p_{k+1}<q_{k+1}$ such that if $c_{j} \in \mathcal{I}\left(O_{\Gamma}\right)$, then $p_{i} \leq j \leq q_{i}$ for some $i=1, \ldots, k+1$.

Let $\eta_{e}: \operatorname{Acyc}(\Gamma) \longrightarrow \operatorname{Acyc}\left(\Gamma^{\prime}\right)$ be the canonical map that sends $O_{\Gamma}$ to $O_{\Gamma^{\prime}}$. This extends naturally to a map $\eta_{e}^{*}: \operatorname{Acyc}(\Gamma) / \sim_{\kappa} \longrightarrow \operatorname{Acyc}\left(\Gamma^{\prime}\right) / \sim_{\kappa}$ between $\kappa$-classes. Define

$$
\mathcal{I}_{e}^{*}: \operatorname{Acyc}\left(\Gamma^{\prime}\right) / \sim_{\kappa} \longrightarrow \operatorname{Acyc}_{\leq}(\Gamma)
$$

by $\mathcal{I}_{e}^{*}\left(\left[O_{\Gamma^{\prime}}\right]\right)=\mathcal{I}\left(O_{\Gamma}^{1}\right)$ for any $O_{\Gamma}^{1} \in\left[O_{\Gamma}\right]$ such that $\eta_{e}^{*}\left(\left[O_{\Gamma}\right]\right)=\left[O_{\Gamma^{\prime}}\right]$ with $\left|\mathcal{I}\left(O_{\Gamma}^{1}\right)\right| \geq 3$, and $\mathcal{I}_{e}^{*}\left(\left[O_{\Gamma^{\prime}}\right]\right)=(v, w)$ (that is, the subgraph induced by $\left.\{v, w\}\right)$ if no such acyclic orientation $O_{\Gamma}^{1}$ exists. The following result relates the $v w$-intervals of $\operatorname{Acyc}(\Gamma) / \sim_{\kappa}$ and $\operatorname{Acyc}\left(\Gamma^{\prime}\right) / \sim_{\kappa}$ through a commutative diagram involving $\mathcal{I}^{*}$ and $\mathcal{I}_{e}^{*}$. An explicit example immediately follows the proof.

Proposition 3.8. The map $\mathcal{I}_{e}^{*}$ is well-defined, and the diagram

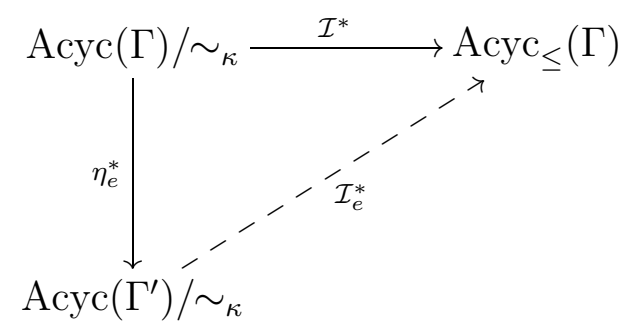

commutes.

Proof. Let $\left[O_{\Gamma^{\prime}}\right] \in \operatorname{Acyc}\left(\Gamma^{\prime}\right) / \sim_{\kappa}$. If there is at most one orientation $O_{\Gamma} \in \operatorname{Acyc}(\Gamma) \operatorname{such}$ that $\left|\mathcal{I}\left(O_{\Gamma}\right)\right| \geq 3$ and $\eta_{e}\left(O_{\Gamma}\right) \in\left[O_{\Gamma^{\prime}}\right]$, or if all orientations of the form $O_{\Gamma}^{1}$ in the definition of $\mathcal{I}_{e}^{*}$ are $\kappa$-equivalent, then both statements of the proposition are clear. Assume therefore that there are acyclic orientations $O_{\Gamma}^{\pi}, O_{\Gamma}^{\sigma} \in \operatorname{Acyc}(\Gamma)$ with $O_{\Gamma}^{\pi} \nsim_{\kappa} O_{\Gamma}^{\sigma}$, but $\eta_{e}^{*}\left(\left[O_{\Gamma}^{\pi}\right]\right)=$ $\eta_{e}^{*}\left(\left[O_{\Gamma}^{\sigma}\right]\right)$ and $\left|\mathcal{I}\left(O_{\Gamma}^{\pi}\right)\right|,\left|\mathcal{I}\left(O_{\Gamma}^{\sigma}\right)\right| \geq 3$. It suffices to prove that in this case,

$$
\mathcal{I}\left(O_{\Gamma}^{\pi}\right)=\mathcal{I}\left(O_{\Gamma}^{\sigma}\right)
$$


This is equivalent to showing that the set of $v w$-paths (directed paths from $v$ to $w$ ) in $O_{\Gamma^{\prime}}^{\pi}$ is the same as the set of $v w$-paths in $O_{\Gamma^{\prime}}^{\sigma}$. From this it will also follow that the diagram commutes. By assumption, both of these orientations contain at least one $v w$-path. We will consider separately the cases when these orientations share or do not share a common vw-path.

Case 1: $O_{\Gamma^{\prime}}^{\pi}$ and $O_{\Gamma^{\prime}}^{\sigma}$ share no common vw-path. Let $P_{1}$ be a length- $k_{1} v w$-path in $O_{\Gamma^{\prime}}^{\pi}$, and let $P_{2}$ be a length- $k_{2} v w$-path in $O_{\Gamma^{\prime}}^{\sigma}$. Suppose that in $O_{\Gamma^{\prime}}^{\pi}$ there are $k_{2}^{+}$edges along $P_{2}$ oriented from $v$ to $w$, and $k_{2}^{-}$edges oriented from $w$ to $v$. Likewise, suppose that in $O_{\Gamma^{\prime}}^{\sigma}$ there are $k_{1}^{+}$edges along $P_{1}$ oriented from $v$ to $w$, and $k_{1}^{-}$edges oriented from $w$ to $v$. If $C=P_{1} P_{2}^{-1}$ (the cycle formed by traversing $P_{1}$ followed by $P_{2}$ in reverse), then

$$
\nu_{C}\left(O_{\Gamma^{\prime}}^{\pi}\right)=k_{1}^{+}+k_{1}^{-}+k_{2}^{-}-k_{2}^{+}, \quad \nu_{C}\left(O_{\Gamma^{\prime}}^{\sigma}\right)=k_{1}^{+}-k_{1}^{-}-k_{2}^{-}-k_{2}^{+} .
$$

Equating these values yields $k_{1}^{-}+k_{2}^{-}=0$, and since these are non-negative integers, $k_{1}^{-}=k_{2}^{-}=0$. We conclude that $P_{1}$ is a $v w$-path in $O_{\Gamma^{\prime}}^{\sigma}$ and $P_{2}$ is a $v w$-path in $O_{\Gamma^{\prime}}^{\pi}$, contradicting the assumption that $O_{\Gamma^{\prime}}^{\pi}$ and $O_{\Gamma^{\prime}}^{\sigma}$ share no common $v w$-paths.

Case 2: $O_{\Gamma^{\prime}}^{\pi}$ and $O_{\Gamma^{\prime}}^{\sigma}$ share a common vw-path $P_{1}$, say of length $k_{1}$. If these are the only $v w$-paths, we are done. Otherwise, assume without loss of generality that $P_{2}$ is another $v w$-path in $O_{\Gamma^{\prime}}^{\pi}$, say of length $k_{2}$. Then if $C=P_{1} P_{2}^{-1}$, we have $\nu_{C}\left(O_{\Gamma^{\prime}}^{\pi}\right)=k_{1}-k_{2}$, and hence $\nu_{C}\left(O_{\Gamma^{\prime}}^{\sigma}\right)=k_{1}-k_{2}$. Therefore, $P_{2}$ is a $v w$-path in $O_{\Gamma^{\prime}}^{\sigma}$ as well. Because $P_{2}$ was arbitrary, we conclude that $O_{\Gamma^{\prime}}^{\pi}$ and $O_{\Gamma^{\prime}}^{\sigma}$ share the same set of $v w$-paths. Since Case 1 is impossible, we have established (6), and the proof is complete.

Example 3.9. Consider the orientations $O_{\Gamma}^{a}, O_{\Gamma}^{b} \in \operatorname{Acyc}(\Gamma)$ from our running example (see Figure 1). Since the vw-intervals of $O_{\Gamma}^{a}$ and $O_{\Gamma}^{b}$ are non-empty (see Example 3.2),

$$
\mathcal{I}^{*}\left(\left[O_{\Gamma}^{a}\right]\right)=\mathcal{I}\left(O_{\Gamma}^{a}\right)=\left\{v, w, v^{\prime}, w^{\prime}\right\}, \quad \mathcal{I}^{*}\left(\left[O_{\Gamma}^{b}\right]\right)=\mathcal{I}\left(O_{\Gamma}^{b}\right)=\{v, w\} .
$$

The natural map $\eta_{e}^{*}$ simply removes the edge $\{v, w\}$, i.e.,

$$
\eta_{e}^{*}\left(\left[O_{\Gamma}^{a}\right]\right)=\left[O_{\Gamma^{\prime}}^{a}\right], \quad \eta_{e}^{*}\left(\left[O_{\Gamma}^{b}\right]\right)=\left[O_{\Gamma^{\prime}}^{b}\right] .
$$

Finally, Proposition 3.8 guarantees a well-defined map $\mathcal{I}_{e}^{*}$ satisfying $\mathcal{I}_{e}^{*} \circ \eta_{e}^{*}=\mathcal{I}^{*}$, and thus

$$
\mathcal{I}_{e}^{*}\left(\left[O_{\Gamma^{\prime}}^{a}\right]\right)=\mathcal{I}^{*}\left(\left[O_{\Gamma}^{a}\right]\right)=\left\{v, w, v^{\prime}, w^{\prime}\right\}, \quad \mathcal{I}_{e}^{*}\left(\left[O_{\Gamma^{\prime}}^{b}\right]\right)=\mathcal{I}^{*}\left(\left[O_{\Gamma}^{b}\right]\right)=\{v, w\} .
$$

This is shown in Figure 2, though note that the domains are the actual $\kappa$-classes containing the given orientations, not the orientations themselves.

Let $O_{\Gamma} \in \operatorname{Acyc}(\Gamma)$ and assume $I=\mathcal{I}\left(O_{\Gamma}\right)$ is non-empty. We write $\Gamma_{I}$ for the graph formed from $\Gamma$ by contracting all vertices in $I$ to a single vertex, which we denote by $V_{I}$. Note that if $I$ only contains $v$ and $w$ then $\Gamma_{I}=\Gamma_{e}^{\prime \prime}$. Moreover, $O_{\Gamma}$ gives rise to an orientation $O_{\Gamma_{I}}$ of $\Gamma_{I}$, and this orientation is clearly acyclic.

Proposition 3.10. Let $O_{\Gamma}^{1}, O_{\Gamma}^{2} \in \operatorname{Acyc}(\Gamma)$ and assume $\mathcal{I}\left(O_{\Gamma}^{1}\right)=\mathcal{I}\left(O_{\Gamma}^{2}\right)$. If $O_{\Gamma}^{1} \nsim_{\kappa} O_{\Gamma}^{2}$ then $\left[O_{\Gamma_{I}}^{1}\right] \nsim_{\kappa}\left[O_{\Gamma_{I}}^{2}\right]$. 


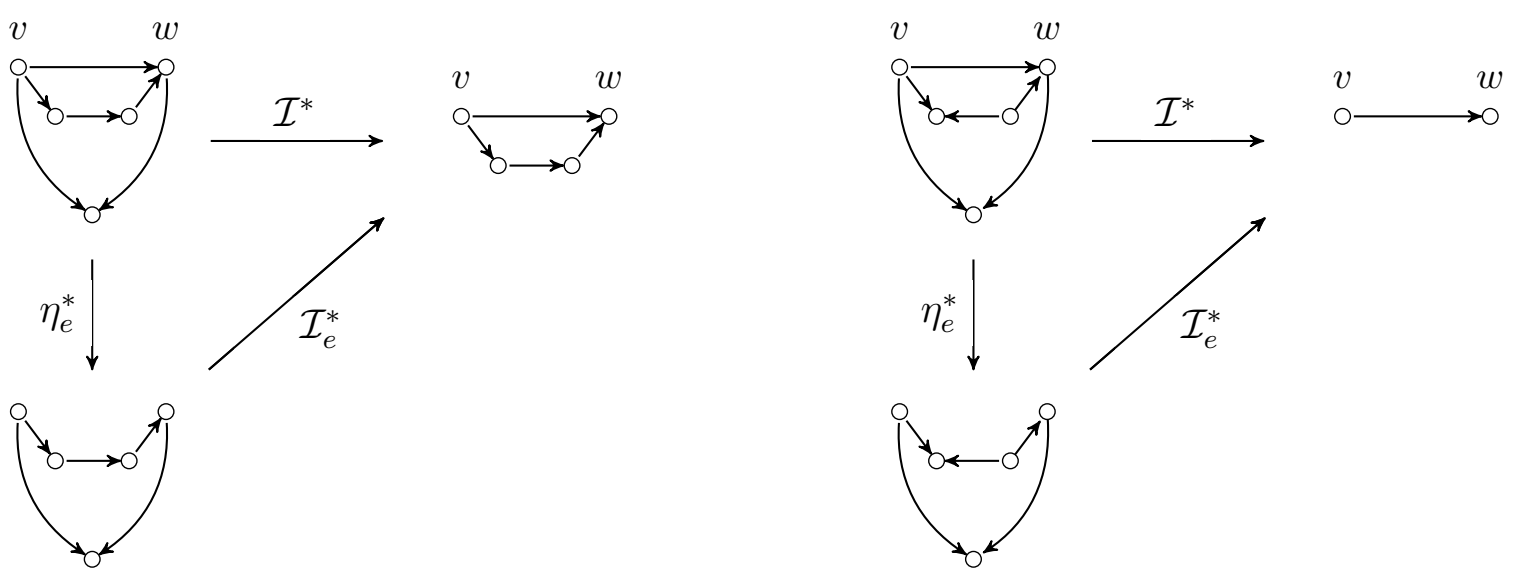

Figure 2: An explicit example of the commutative diagram relating the maps $\eta_{e}^{*}, \mathcal{I}^{*}$, and $\mathcal{I}_{e}^{*}$ from Proposition 3.8. The domain of these maps are actually the sets of $\kappa$-equivalence classes, e.g., $\left[O_{\Gamma}^{a}\right]$ (left) and $\left[O_{\Gamma}^{b}\right]$ (right), but they are shown acting on actual orientations $\left(O_{\Gamma}^{a}\right.$ and $\left.O_{\Gamma}^{b}\right)$ for clarity.

Proof. We prove the contrapositive statement. Set $I=\mathcal{I}\left(O_{\Gamma}^{1}\right)$, suppose $|I|=k$, and let $v_{1} v_{2} \cdots v_{k}$ be a linear extension of $I$. For any click-sequence $\mathbf{c}_{I}$ between two acyclic orientations $O_{\Gamma_{I}}^{1}$ and $O_{\Gamma_{I}}^{2}$ in $\operatorname{Acyc}\left(\Gamma_{I}\right)$, let $\mathbf{c}$ be the click-sequence formed by replacing every occurrence of $V_{I}$ in $\mathbf{c}_{I}$ by the sequence $v_{1} \cdots v_{k}$. Then $\mathbf{c}\left(O_{\Gamma}^{1}\right)=O_{\Gamma}^{2}$ and $O_{\Gamma}^{1} \sim_{\kappa} O_{\Gamma}^{2}$ as claimed.

We can now utilize the results on poset structure just developed to establish a bijection

$$
\Theta: \operatorname{Acyc}(\Gamma) / \sim_{\kappa} \longrightarrow\left(\operatorname{Acyc}\left(\Gamma_{e}^{\prime}\right) / \sim_{\kappa} \cup \operatorname{Acyc}\left(\Gamma_{e}^{\prime \prime}\right) / \sim_{\kappa}\right)
$$

valid for any cycle-edge $e$. For $\left[O_{\Gamma}\right] \in \operatorname{Acyc}(\Gamma) / \sim_{\kappa}$, let $O_{\Gamma}^{\pi}$ denote an orientation in $\left[O_{\Gamma}\right]$ such that $\pi=v \pi_{2} \cdots \pi_{n}$ and $w=\pi_{i}$ for $i$ minimal. We define $\Theta$ by

$$
\left[O_{\Gamma}\right] \stackrel{\Theta}{\longmapsto} \begin{cases}{\left[O_{\Gamma^{\prime \prime}}^{\pi}\right],} & \exists O_{\Gamma}^{\pi} \in\left[O_{\Gamma}\right] \text { with } \pi=v w \pi_{3} \cdots \pi_{n} \\ {\left[O_{\Gamma^{\prime}}^{\pi}\right],} & \text { otherwise. }\end{cases}
$$

Note that $\left[O_{\Gamma}\right]$ is mapped into $\operatorname{Acyc}\left(\Gamma^{\prime \prime}\right) / \sim_{\kappa}$ if and only if the only vertices in $\mathcal{I}_{e}^{*}\left(\left[O_{\Gamma}\right]\right)$ are $v$ and $w$. Since $\kappa$-equivalence over $\Gamma$ implies $\kappa$-equivalence over $\Gamma^{\prime}, \Theta$ does not depend on the choice of $\pi$, and thus is well-defined. We continue our running example below to illustrate this.

Example 3.11. The orientations $O_{\Gamma}^{a}$ and $O_{\Gamma}^{b}$ from our running example are shown in Figure 3. Since $\mathcal{I}_{e}^{*}\left(\left[O_{\Gamma}^{a}\right]\right)=\left\{v, v^{\prime}, w^{\prime}, w\right\}$, the map $\Theta$ removes the edge $e=\{v, w\}$. However, since $\mathcal{I}_{e}^{*}\left(\left[O_{\Gamma}^{b}\right]\right)=\{v, w\}$, the map $\Theta$ contracts $e$.

The results we have derived for the $v w$-interval now allow us to establish the following:

Theorem 3.12. The map $\Theta$ is a bijection. 


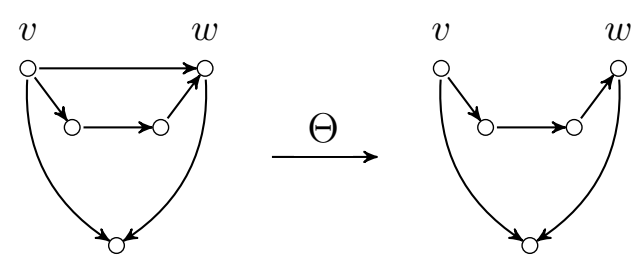

(a) $O_{\Gamma}^{a} \stackrel{\Theta}{\longmapsto} O_{\Gamma^{\prime}}^{a}$

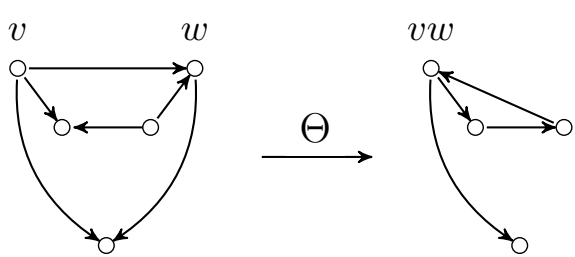

(b) $O_{\Gamma}^{b} \stackrel{\Theta}{\longmapsto} O_{\Gamma^{\prime \prime}}^{b}$

Figure 3: An example of the map $\Theta$ applied to the orientations $O_{\Gamma}^{a}$ and $O_{\Gamma}^{b}$ from Example 2.1 and Figure 1. If contracting the edge $e=\{v, w\}$ would introduce a directed cycle (as in $O_{\Gamma}^{a}$ ), then we must delete it. Otherwise (as in $O_{\Gamma}^{b}$ ), contract it. Note that this happens precisely when $(v, w)$ is the only directed path from $v$ to $w$.

Proof. We first prove that $\Theta$ is surjective. Let $I=\{v, w\}$ and consider an element $\left[O_{\Gamma^{\prime \prime}}\right] \in$ $\operatorname{Acyc}\left(\Gamma^{\prime \prime}\right) / \sim_{\kappa}$ with $O_{\Gamma^{\prime \prime}}^{\pi} \in\left[O_{\Gamma^{\prime \prime}}\right]$ where $\pi=V_{I} \pi_{2} \cdots \pi_{n-1}$. Let $\pi^{+}=v w \pi_{2} \cdots \pi_{n-1} \in S_{\Gamma}$. Clearly $\left[O_{\Gamma}^{\pi^{+}}\right] \in \operatorname{Acyc}(\Gamma) / \sim_{\kappa}$ is mapped to $\left[O_{\Gamma^{\prime \prime}}\right]$ by $\Theta$.

Next, consider an element $\left[O_{\Gamma^{\prime}}\right] \in \operatorname{Acyc}\left(\Gamma^{\prime}\right) / \sim_{\kappa}$. If there is no element $O_{\Gamma^{\prime}}^{\pi}$ of $\left[O_{\Gamma^{\prime}}\right]$ such that $\pi=v w \pi_{3} \cdots \pi_{n}$, then no elements of $\left[O_{\Gamma}\right]$ are of this form either, and by definition $\left[O_{\Gamma^{\prime}}\right]$ has a preimage under $\Theta$. We are left with the case where $\left[O_{\Gamma^{\prime}}\right]$ contains an element $O_{\Gamma^{\prime}}^{\pi}$ such that $\pi=v w \pi_{3} \cdots \pi_{n}$, and we must show that there exists $O_{\Gamma^{\prime}}^{\pi^{\prime}} \in\left[O_{\Gamma^{\prime}}\right]$ such that $\left[O_{\Gamma}^{\pi^{\prime}}\right]$ contains no element of the form $O_{\Gamma}^{\sigma}$ with $\sigma=v w \sigma_{3} \cdots \sigma_{n}$. Note that if $\sigma=v w \sigma_{3} \cdots \sigma_{n}$, then the vertices in $\mathcal{I}\left(O_{\Gamma}^{\sigma}\right)$ are precisely $v$ and $w$. If the orientation $O_{\Gamma^{\prime}}$ had a directed path from $v$ to $w$, then the corresponding orientation $O_{\Gamma} \in \operatorname{Acyc}(\Gamma)$ formed by adding the edge $e$ with orientation $(v, w)$ has $v w$-interval of size at least 3 , so by Proposition 3.3, the acyclic orientation $O_{\Gamma}$ cannot be $\kappa$-equivalent to any orientation $O_{\Gamma}^{\sigma}$ such that $\sigma=v w \sigma_{3} \cdots \sigma_{n}$.

Thus it remains to consider the case when $\left[O_{\Gamma^{\prime}}\right]$ contains no acyclic orientation with a directed path from $v$ to $w$. Pick any simple undirected path $P^{\prime}$ from $v$ to $w$ in $\Gamma^{\prime}$, which is possible since $e$ is a cycle-edge. Choose an orientation in $\left[O_{\Gamma^{\prime}}\right]$ for which $\nu_{P^{\prime}}$ is maximal. Without loss of generality we may assume that $O_{\Gamma^{\prime}}$ is this orientation. Let $O_{\Gamma} \in \operatorname{Acyc}(\Gamma)$ be the orientation that agrees with $O_{\Gamma^{\prime}}$, and with $e$ oriented as $(w, v)$. Since we have assumed that there is no directed path from $v$ to $w$ this orientation is acyclic. We claim that for any $\sigma=v w \sigma_{3} \cdots \sigma_{n}$ one has $O_{\Gamma}^{\sigma} \notin\left[O_{\Gamma}\right]$. To see this, assume the statement is false. Let $P$ be the undirected cycle in $\Gamma$ formed by adding the edge $e$ to the path $P^{\prime}$. Because $e$ is oriented as $(v, w)$ in $O_{\Gamma}^{\sigma}$ and as $(w, v)$ in $O_{\Gamma}$, we have $\nu_{P}\left(O_{\Gamma}^{\sigma}\right)=\nu_{P^{\prime}}\left(O_{\Gamma^{\prime}}^{\sigma}\right)-1$ and $\nu_{P}\left(O_{\Gamma}\right)=\nu_{P^{\prime}}\left(O_{\Gamma^{\prime}}\right)+1$. If $O_{\Gamma}$ and $O_{\Gamma}^{\sigma}$ were $\kappa$-equivalent, then

$$
\nu_{P^{\prime}}\left(O_{\Gamma^{\prime}}^{\sigma}\right)-1=\nu_{P}\left(O_{\Gamma}^{\sigma}\right)=\nu_{P}\left(O_{\Gamma}\right)=\nu_{P^{\prime}}\left(O_{\Gamma}\right)+1,
$$

and thus $\nu_{P^{\prime}}\left(O_{\Gamma^{\prime}}^{\sigma}\right)=\nu_{P^{\prime}}\left(O_{\Gamma}\right)+2$. Any click sequence mapping $O_{\Gamma}$ to $O_{\Gamma}^{\sigma}$ is a click-sequence from $O_{\Gamma^{\prime}}$ to $O_{\Gamma^{\prime}}^{\sigma}$. Therefore, $O_{\Gamma^{\prime}}^{\sigma} \in\left[O_{\Gamma^{\prime}}\right]$, which contradicts the maximality of $\nu_{P^{\prime}}\left(O_{\Gamma^{\prime}}\right)$. We therefore conclude that $O_{\Gamma}^{\sigma} \notin\left[O_{\Gamma}\right]$, that $\Theta\left(\left[O_{\Gamma}\right]\right)=\left[O_{\Gamma^{\prime}}\right]$, and hence that $\Theta$ is surjective.

We next prove that $\Theta$ is an injection. By Proposition 3.10 (with $I=\{v, w\}$ ), $\Theta$ is injective when restricted to the preimage of $\left[O_{\Gamma^{\prime \prime}}\right]$ under $\Theta$. Thus it suffices to show 
that every element in $\operatorname{Acyc}\left(\Gamma^{\prime}\right) / \sim_{\kappa}$ has a unique preimage under $\Theta$. By Proposition 3.8, every preimage of $\left[O_{\Gamma^{\prime}}\right]$ must have the same $v w$-interval $I$, containing $k>2$ vertices. Suppose there were preimages $\left[O_{\Gamma}^{\pi}\right] \neq\left[O_{\Gamma}^{\sigma}\right]$ of $\left[O_{\Gamma^{\prime}}\right]$. By Proposition 3.10, it follows that $O_{\Gamma_{I}}^{\pi} \nsim_{\kappa} O_{\Gamma_{I}}^{\sigma}$. We will now show that this leads to a contradiction.

Assume that $\mathbf{c}=c_{1} \cdots c_{m}$ is a click-sequence from $O_{\Gamma^{\prime}}^{\pi}$ to $O_{\Gamma^{\prime}}^{\sigma}$. If one of $\pi$ or $\sigma$ is not $\kappa^{-}$ equivalent to a permutation with vertices $v$ and $w$ in succession, then their corresponding $\kappa$-classes would be unchanged by the removal of edge $e$. In light of this, we may assume that $\pi=v \pi_{2} \ldots \pi_{n-1} w$ and $\sigma=v \sigma_{2} \ldots \sigma_{n-1} w$, and thus that $c_{1}=v$ and $c_{m}=w$. By Proposition 3.6, we may assume that the vertices in $I$ appear in $\mathbf{c}$ in some number of disjoint consecutive "blocks," i.e., subsequences of the form $c_{i} \cdots c_{i+k-1}$. Replacing each of these blocks with $V_{I}$ yields a click-sequence from $O_{\Gamma_{I}}^{\pi}$ to $O_{\Gamma_{I}}^{\sigma}$, contradicting the fact that $O_{\Gamma_{I}}^{\pi} \nsim_{\kappa} O_{\Gamma_{I}}^{\sigma}$. Therefore, no such click sequence $\mathbf{c}$ exists, and $\Theta$ must be an injection, and the proof is complete.

The main result in [10] is a recurrence relation for $\kappa(\Gamma)$ under edge deletion and edge contraction. This is an easy consequence of Theorem 3.12.

Corollary 3.13 ([10]). Let $\Gamma$ be a finite undirected graph with e $\in \mathrm{e}[\Gamma]$, and let $\Gamma_{e}^{\prime}$ be the graph obtained from $\Gamma$ by deleting $e$, and let $\Gamma_{e}^{\prime \prime}$ be the graph obtained from $\Gamma$ by contracting e. Then

$$
\kappa(\Gamma)=\left\{\begin{aligned}
\kappa\left(\Gamma_{1}\right) \kappa\left(\Gamma_{2}\right), & e \text { is a bridge linking } \Gamma_{1} \text { and } \Gamma_{2}, \\
\kappa\left(\Gamma_{e}^{\prime}\right)+\kappa\left(\Gamma_{e}^{\prime \prime}\right), & e \text { is a cycle-edge }
\end{aligned}\right.
$$

The first part involving a bridge is straightforward, while the second part is a direct consequence of Theorem 3.12.

\section{A Complete Invariant of $\operatorname{Acyc}(\Gamma) / \sim_{\kappa}$}

Theorem 3.12 is more than just an alternative proof of the enumeration of $\kappa(\Gamma)$. We can utilize the explicit bijection to derive an additional interesting and useful corollary: When taken over all cycles $C$ in a graph $\Gamma, \nu_{C}$ is a complete invariant of $\operatorname{Acyc}(\Gamma) / \sim_{\kappa}$. This result is originally due to Pretzel [16], though the techniques are much different than the ones here.

Theorem 4.1. If $\nu_{C}\left(O_{\Gamma}^{1}\right)=\nu_{C}\left(O_{\Gamma}^{2}\right)$ for every cycle $C$, then $O_{\Gamma}^{1} \sim_{\kappa} O_{\Gamma}^{2}$.

Proof. Assume the statement is false and let $\Gamma$ be a graph for which it fails, minimal with respect to $|\mathrm{e}[\Gamma]|$. Fix a cycle-edge $e=\{v, w\}$, and for any $\left[O_{\Gamma}\right] \in \operatorname{Acyc}(\Gamma) / \sim_{\kappa}$, call an orientation $O_{\Gamma}^{\pi} \in\left[O_{\Gamma}\right]$ distinguished with respect to $e$ if $\pi=\pi_{1} \pi_{2} \cdots \pi_{n}$ such that $(i)$ $\pi_{1}=v$, and $(i i) \pi_{k}=w$ where $k$ is minimal given that $\pi_{1}=v$. By assumption, there exists $O_{\Gamma}^{1} \nsim_{\kappa} O_{\Gamma}^{2}$ with $\nu_{C}\left(O_{\Gamma}^{1}\right)=\nu_{C}\left(O_{\Gamma}^{2}\right)$ for every cycle $C$ in $\Gamma$. Without loss of generality, we may assume that $O_{\Gamma}^{1}$ and $O_{\Gamma}^{2}$ are distinguished orientations with respect to $e=\{v, w\}$. Define a $v w$-path to be a directed path from $v$ to $w$ that does not traverse $e$. There are three cases to consider: 
Case 1: Both $O_{\Gamma}^{1}$ and $O_{\Gamma}^{2}$ contain a vw-path. By definition of $\Theta$, both $\Theta\left(O_{\Gamma}^{1}\right)$ and $\Theta\left(O_{\Gamma}^{2}\right)$ are contained in $\operatorname{Acyc}\left(\Gamma_{e}^{\prime}\right) / \sim_{\kappa}$. Moreover, because $\Theta$ is a bijection, they are distinct elements in $\operatorname{Acyc}\left(\Gamma_{e}^{\prime}\right) / \sim_{\kappa}$. Every cycle in $\Gamma_{e}^{\prime}$ is also a cycle in $\Gamma$, and therefore, $\nu_{C^{\prime}}\left(\Theta\left(O_{\Gamma}^{1}\right)\right)=\nu_{C^{\prime}}\left(\Theta\left(O_{\Gamma}^{2}\right)\right)$ for every cycle $C^{\prime}$ in $\Gamma_{e}^{\prime}$, contradicting the minimality of $|\mathrm{e}[\Gamma]|$.

Case 2: Neither $O_{\Gamma}^{1}$ nor $O_{\Gamma}^{2}$ contain a vw-path. Since $O_{\Gamma}^{1}$ and $O_{\Gamma}^{2}$ are distinguished with respect to $e, \Theta\left(\left[O_{\Gamma}^{i}\right]\right)=\left[O_{\Gamma^{\prime \prime}}^{i}\right] \in \operatorname{Acyc}\left(\Gamma^{\prime \prime}\right) / \sim_{\kappa}$ for $i=1,2$. Again, these two orientations are distinct because $\Theta$ is a bijection. Any cycle $C^{\prime \prime}$ in $\Gamma_{e}^{\prime \prime}$ beginning and ending at the vertex $V$ (the image of $v$ and $w$ under contraction) can be canonically extended to a cycle $C$ of $\Gamma$. Therefore, $\nu_{C^{\prime \prime}}\left(\Theta\left(O_{\Gamma^{\prime \prime}}^{1}\right)\right)=\nu_{C^{\prime \prime}}\left(\Theta\left(O_{\Gamma^{\prime \prime}}^{2}\right)\right)$, again contradicting the minimality of $|\mathrm{e}[\Gamma]|$.

Case 3: Precisely one of $O_{\Gamma}^{1}$ and $O_{\Gamma}^{2}$ contain a vw-path. Without loss of generality, suppose that $P$ is a length- $k v w$-path in $O_{\Gamma}^{1}$, and let $C$ be the cycle formed by adding vertex $v$ to the end of $P$. Clearly, $\nu_{C}\left(O_{\Gamma}^{1}\right)=k-1$, and by assumption, $\nu_{C}\left(O_{\Gamma}^{2}\right)=k-1$ as well. However, this means that every edge in $P$ is oriented from $v$ to $w$ in $O_{\Gamma}^{2}$, contradicting the assumption that $O_{\Gamma}^{2}$ did not contain a $v w$-path. Therefore, Case 3 is impossible, and the proof is complete.

Example 4.2. It is immediate from the recurrence (8) in Corollary 3.13 that $\kappa(\Gamma)=1$ if and only if $\Gamma$ is a forest. Let Circle $_{n}$ be a chordless $n$-cycle, and Line $_{n}$ the line graph on $n$ vertices. Deleting or contracting any edge of $\mathrm{Circle}_{3}$ leaves a tree, and so $\kappa\left(\mathrm{Circle}_{3}\right)=2$. For $n>3$, deleting an edge from Circle $_{n}$ leaves Line $_{n}$, and contracting an edge leaves Circle $_{n-1}$. By Corollary 3.13, if $n>3$, then

$$
\kappa\left(\text { Circle }_{n}\right)=\kappa\left(\text { Line }_{n}\right)+\kappa\left(\text { Circle }_{n-1}\right)=1+\kappa\left(\text { Circle }_{n-1}\right) .
$$

From the base case of $\kappa\left(\right.$ Circle $\left._{3}\right)=2$, we immediately deduce that $\kappa\left(\right.$ Circle $\left._{n}\right)=n-1$.

Example 4.3. Let $\Gamma$ be the undirected version of the orientations from our running example, with edge $e=\{v, w\}$ as before. Deleting e leaves $\Gamma_{e}^{\prime}$, a 5-cycle. Contracting e leaves $\Gamma_{e}^{\prime \prime}$, a 3-cycle with an extra edge hanging off (see Figure 3 in Example 3.11). By the recurrence (8) in Corollary 3.13 along with (9),

$$
\kappa(\Gamma)=\kappa\left(\Gamma_{e}^{\prime}\right)+\kappa\left(\Gamma_{e}^{\prime \prime}\right)=4+2=6 .
$$

Representatives from the six distinct $\kappa$-classes of $\Gamma$ are shown in Figure 4 . This particular transversal was chosen so that $v$ is a source, and so the vw-intervals can be identified immediately, and they are (from left-to-right)

$$
\left\{v, v^{\prime}, w^{\prime}, w\right\}, \quad\{v, w\}, \quad\left\{v, v^{\prime}, w^{\prime}, z, w\right\}, \quad\{v, z, w\}, \quad\{v, z, w\}, \quad\{v, w\} .
$$

Note that the first two orientations in Figure 4 are $O_{\Gamma}^{a}$ and $O_{\Gamma}^{b}$, and the third is $\kappa$-equivalent to $O_{\Gamma}^{c}$. Letting $P=v, v^{\prime}, w^{\prime}, w, v$ and $Q=v, z, w, v$ be the paths as defined in Example 3.2, the pair $\left(\nu_{P}, \nu_{Q}\right)$ is a complete invariant of $\operatorname{Acyc}(\Gamma) / \sim_{\kappa}$. For each of the six orientations, the values of $\nu_{P}\left(O_{\Gamma}\right)$ (top) and $\nu_{Q}\left(O_{\Gamma}\right)$ (bottom) are shown in Figure 4. 

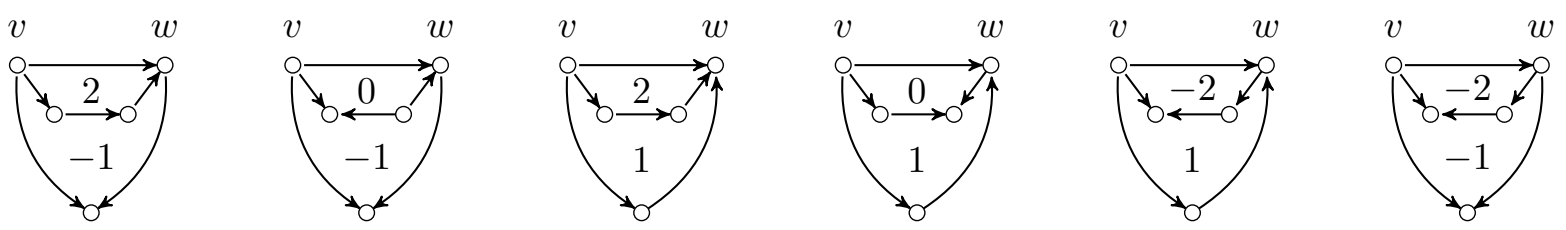

Figure 4: A transversal for $\operatorname{Acyc}(\Gamma) / \sim_{\kappa}$, with the values of the complete invariant $\left(\nu_{P}, \nu_{Q}\right)$.

\section{Conjugacy of Coxeter Elements}

Our analysis of $\operatorname{Acyc}(\Gamma) / \sim_{\kappa}$ also gives a straightforward solution to the conjugacy problem for Coxeter elements in simply-laced Coxeter groups. Before stating the theorem and proof, we will briefly review the connection between $\kappa$-equivalence and Coxeter theory, as described in [10]. A Coxeter group is a generalized reflection group, generated by $n$ distinguished involutions $s_{1}, \ldots, s_{n}$ by the presentation

$$
W=\left\langle s_{1}, \ldots, s_{n} \mid\left(s_{i} s_{j}\right)^{m_{i j}}\right\rangle,
$$

where $m_{i j}=1$ iff $i=j$, and $m_{i j} \geq 2$ otherwise. If $s_{i} s_{j}$ has infinite order, then we say that $m_{i j}=\infty$. The pair $(W, S)$ of the group $W$ with the generating set $S$ is called a Coxeter system, which is uniquely encoded by its Coxeter graph $\Gamma$, with vertex set $S$ and edge set $\left\{s_{i}, s_{j}\right\}$ for which $m_{i j} \geq 3$, where each edge has weight $m_{i j}$. A Coxeter group is simply-laced if each $m_{i j} \leq 3$, thus every undirected graph is the Coxeter graph of a simply-laced Coxeter group.

A Coxeter element is the product of the generators in some order, and there is a natural bijection between the set $\mathrm{C}(W, S)$ of Coxeter elements $\prod_{i} s_{\pi(i)}$ of a Coxeter group (see, e.g. [2]) with generators $s_{i}$ for $1 \leq i \leq n$ and Coxeter graph $\Gamma$ (ignoring bond strengths), and the set of $\alpha$-equivalence classes $[\pi]_{\Gamma}$. This is clear since the commuting generators are precisely those that are not connected in $\Gamma$. Thus, there is a natural bijection

$$
\mathrm{C}(W, S) \longrightarrow \operatorname{Acyc}(\Gamma) .
$$

Moreover, conjugating a Coxeter element $c=\prod s_{\pi(i)}$ by $s_{\pi(1)}$ corresponds to a cyclic shift, i.e.,

$$
s_{\pi(1)}\left(s_{\pi(1)} s_{\pi(2)} \cdots s_{\pi(n)}\right) s_{\pi(1)}=s_{\pi(2)} \cdots s_{\pi(n)} s_{\pi(1)},
$$

since each generator $s_{i}$ is an involution. Therefore, $\kappa$-equivalence naturally carries over to an equivalence relation on $\mathrm{C}(W, S)$. The $\nu$-function carries over as well - define $\nu_{P}(c)$ to be $\nu_{P}\left(O_{\Gamma}\right)$, where $O_{\Gamma}$ is the acyclic orientation of $\Gamma$ corresponding to $c$. It is now elementary to see that $c, c^{\prime} \in \mathrm{C}(W, S)$ are conjugate if $c \sim_{\kappa} c^{\prime}$. However, the converse of this statement was not proven until 2009.

Theorem $5.1([6])$. Let $(W, S)$ be a Coxeter system. Then two Coxeter elements $c, c^{\prime} \in$ $\mathrm{C}(W, S)$ are conjugate if and only if $c \sim_{\kappa} c^{\prime}$.

It follows immediately that the number of distinct conjugacy classes containing Coxeter elements is exactly $\kappa(\Gamma)$. Until the Erikssons' proof, the result was known only for 
the special case of $\mathrm{C}(W, S)$ when $\Gamma$ was simply-laced and unicyclic, established by Shi in 2001 [18]. It is elementary to weaken the simply-laced condition to the bond strengths being multiples of three or infinite, which Shi mentions in [18]. The bijection in Theorem 3.12 applied to Shi's result for unicyclic Coxeter graphs yields a simple and elegant proof of the result for all simply-laced systems, which we present below.

Proof. Suppose for sake of contradiction that the Coxeter elements $c=c_{1} c_{2} \cdots c_{n}$ and $c^{\prime}=c_{1}^{\prime} c_{2}^{\prime} \cdots c_{n}^{\prime}$ are conjugate with $c \nsim_{\kappa} c^{\prime}$, and that $w c w^{-1}=c^{\prime}$ for some $w=w_{1} \ldots w_{k} \in$ $W$ with each $w_{i} \in S$. By Theorem 4.1, there is some simple chordless cycle $P=$ $v_{0}, v_{1}, \ldots, v_{m-1}, v_{m}$ (i.e., $\left.v_{0}=v_{m}\right)$ in $\Gamma$ such that $\nu_{P}(c) \neq \nu_{P}\left(c^{\prime}\right)$. Let $S_{P}=\left\{s_{i} \mid i \in P\right\}$, and let $C_{m}$ be the (circular) Coxeter graph induced by the vertices in $P$. The Coxeter group generated by $S_{P}$ is the affine Weyl group $\widetilde{A}_{m-1}$, and there is a natural homomorphism $W \stackrel{\varphi}{\longrightarrow} \widetilde{A}_{m-1}$ defined on the generators by

$$
s_{i} \stackrel{\varphi}{\longmapsto}\left\{\begin{array}{cc}
s_{i} & i \in P \\
1 & i \notin P .
\end{array}\right.
$$

Since $c$ and $c^{\prime}$ are conjugate in $W, \varphi(c)$ and $\varphi\left(c^{\prime}\right)$ are conjugate in $\widetilde{A}_{m-1}$. By choice of $P$, $\nu_{P}(c) \neq \nu_{P}\left(c^{\prime}\right)$, and thus $\varphi(c) \nsim_{\kappa} \varphi\left(c^{\prime}\right)$. However, since the statement holds for unicyclic graphs, we must have $\varphi(c) \sim_{\kappa} \varphi\left(c^{\prime}\right)$, which is the desired contradiction.

Theorems 4.1 and 5.1 give us an easy way to verify whether two Coxeter elements $c$ and $c^{\prime}$ in any Coxeter group $W$ are conjugate. Pick a cycle basis of the Coxeter graph $\Gamma$, and for each cycle $C$, compute $\nu_{C}(c)$ and $\nu_{C}\left(c^{\prime}\right)$. By Theorem 4.1, $c \sim_{\kappa} c^{\prime}$ iff $\nu_{C}(c)=\nu_{C}\left(c^{\prime}\right)$ for each $C$. By Theorem 5.1, this is equivalent to $c$ and $c^{\prime}$ being conjugate in $W$. Therefore, conjugacy of Coxeter elements can be verified in $O\left(n^{2}\right)$ steps, where $n=|\mathrm{v}[\Gamma]|$. One application of this is seeing how the conjugacy classes split as an edge $\left\{s_{i}, s_{j}\right\}$ is added to $\Gamma$ (or equivalently, as the relation $\left(s_{i} s_{j}\right)^{m_{i j}}$ is added to the group presentation).

\section{Discrete Dynamical Systems, Node-firing Games, and Quiver Representations}

We conclude with a brief discussion of how the equivalence relation studied in this paper arises in various areas of mathematics. The original motivation came from both authors' interest in sequential dynamical systems (SDSs). The equivalence relation $\sim_{\alpha}$ arises naturally in the study of functional equivalence of these systems. This can be seen as follows. Given a graph $\Gamma$ with vertex set $\{1,2, \ldots, n\}$ as above, a state $x_{v} \in K$ is assigned to each vertex $v$ of $\Gamma$ for some finite set $K$. The system state is the tuple consisting of all the vertex states, and is denoted by $x=\left(x_{1}, \ldots, x_{n}\right) \in K^{n}$. The sequence of states associated to the 1-neighborhood $B_{1}(v ; \Gamma)$ of $v$ in $\Gamma$ (in some fixed order) is denoted by $x[v]$. A sequence of vertex functions $\left(f_{i}\right)_{i}$ with $f_{i}: K^{d(i)+1} \longrightarrow K$ induces $\Gamma$-local functions $F_{i}: K^{n} \longrightarrow K^{n}$ of the form

$$
F_{i}\left(x_{1}, \ldots, x_{n}\right)=\left(x_{1}, \ldots, x_{i-1}, f_{i}(x[i]), x_{i+1}, \ldots, x_{n}\right) .
$$


The sequential dynamical system map with update order $\pi=\left(\pi_{i}\right)_{i} \in S_{\Gamma}$ is the function composition

$$
F_{\pi}=F_{\pi_{n}} \circ F_{\pi_{n-1}} \circ \cdots \circ F_{\pi_{2}} \circ F_{\pi_{1}} .
$$

By construction, if $\pi \sim_{\alpha} \pi^{\prime}$ holds, then $F_{\pi}$ and $F_{\pi^{\prime}}$ are identical as functions, independent of the choice of state space $K$ or vertex functions. Thus, $\alpha(\Gamma)$ is a general upper bound for the number of functionally non-equivalent SDS maps that can be generated over the graph $\Gamma$ for a fixed sequence of $\Gamma$-local functions. Moreover, for any graph $\Gamma$, there exist $\Gamma$-local functions for which this bound is sharp [14]. A weaker form of equivalence is cycle equivalence, which means that the dynamical system maps are conjugate (using the discrete topology) when restricted to their sets of periodic points. In the language of graph theory, this means their periodic orbits are isomorphic as directed graphs. For an update order $\pi=\pi_{1} \cdots \pi_{n}$, define $\operatorname{shift}(\pi)=\pi_{2} \cdots \pi_{n} \pi_{1}$. The following result shows how $\kappa$-equivalent update orders yield dynamical system maps that are cycle equivalent.

Theorem 6.1. For any finite set $K$ of vertex states, and for any $\pi \in S_{\Gamma}$, the $S D S$ maps $F_{\pi}$ and $F_{\text {shift }(\pi)}$ are cycle equivalent.

We refer to [11] for the proof of this result, as well as additional background on equivalences of sequential dynamical systems, and applications of $\kappa$-equivalence to the structural properties of their phase spaces. It is interesting to note that for the class of update sequence independent (see [9]) sequential dynamical systems with binary states, there is an additional equivalence on acyclic orientations that governs cycle equivalence: reversal of all edge orientations.

The chip-firing game was introduced by Björner, Lovász, and Shor [3]. It is played over an undirected graph $\Gamma$, and each vertex is given some number of (but possibly zero) chips. If vertex $i$ has degree $d_{i}$, and at least $d_{i}$ chips, then a legal move (or a "click") of vertex $i$ is a transfer of one chip to each neighboring vertex. This may be viewed as a generalization of a source-to-sink move for acyclic orientations where the out-degree of a vertex plays the role of the chip count. The chip-firing game is closely related to the numbers game [2]. In the numbers game over a graph $\Gamma$, the legal sequences of moves are in 1-1 correspondence with the reduced words of the Coxeter group with Coxeter graph $\Gamma$. For an excellent summary and comparison of these games, see [7].

A quiver is a finite directed graph (loops and multiple edges are allowed), and appears primarily in the study of representation theory. A quiver $Q$ with a field $K$ gives rise to a path algebra $K Q$, and there is a natural correspondence between $K Q$-modules and $K$-representations of $Q$. In fact, there is an equivalence between the categories of quiver representations, and modules over path algebras. A path algebra is finite-dimensional if and only if the quiver is acyclic, and the modules over finite-dimensional path algebras form a reflective subcategory. A reflection functor maps representations of a quiver $Q$ to representations of a quiver $Q^{\prime}$, where $Q^{\prime}$ differs from $Q$ by a source-to-sink operation [12]. We note that while the composition of $n$ source-to-sink operations (one for each vertex) maps a quiver back to itself, the corresponding composition of reflection functors is not 
the identity, but rather a Coxeter functor. In fact, the same result in [19] about powers of Coxeter elements being reduced was proven previously using techniques from the representation theory of quivers [8].

We hope this paper will motivate further explorations of the connections between these topics. We are particularly curious about any implications to the representation theory of quivers. This is a field which the both authors of this paper are quite unfamiliar with, yet it motivated Kleiner and Pelley to study admissible sequences and apply these tools from quiver representations to Coxeter groups. Without this work, the aforementioned papers of Speyer and the Erikssons would likely not have materialized.

Acknowledgments. Both authors are grateful to the NDSSL group at Virginia Tech for the support of this research. Special thanks to Ed Green and Ken Millett for helpful discussions and feedback, and to William Y. C. Chen for valuable advice regarding the preparation and structuring of this paper.

\section{References}

[1] M. Auslander, I. Reiten, and S. O. Smalø. Representation Theory of Artin Algebras. Cambridge University Press, 1997.

[2] A. Björner and F. Brenti. Combinatorics of Coxeter Groups. Springer-Verlag, New York, 2005.

[3] A. Björner, L. Lovász, and P. Shor. Chip-firing games on graphs. European J. Combin., 12:283-291, 1991.

[4] P. Cartier and D. Foata. Problemes combinatoires de commutation et reárrangements, volume 85 of Lecture Notes in Mathematics. Springer Verlag, 1969.

[5] A. J. Coleman. Killing and the Coxeter transformation of Kac-Moody algebras. Invent. Math., 95:447-477, 1989.

[6] H. Eriksson and K. Eriksson. Conjugacy of Coxeter elements. Electron. J. Combin., 16(2):\#R4, 2009.

[7] K. Eriksson. Node firing games on graphs. Contemp. Math., 178:117-127, 1994.

[8] M. Kleiner and A. Pelley. Admissible sequences, preprojective representations of quivers, and reduced words in the Weyl group of a Kac-Moody algebra. Internat. Math. Res. Notices, 2007, May 2007.

[9] M. Macauley, J. McCammond, and H. S. Mortveit. Dynamics groups of asynchronous cellular automata. J. Algebraic Combin., 33:31-55, 2011.

[10] M. Macauley and H. S. Mortveit. On enumeration of conjugacy classes of Coxeter elements. Proc. Amer. Math. Soc., 136:4157-4165, 2008. 
[11] M. Macauley and H. S. Mortveit. Cycle equivalence of graph dynamical systems. Nonlinearity, 22:421-436, 2009.

[12] R. Marsh, M. Reineke, and A. Zelevinsky. Generalized associahedra via quiver representations. Trans. Amer. Math. Soc., 355:4171-4186, 2003.

[13] E. Miller, V. Reiner, and B. Sturmfels, editors. Geometric Combinatorics, volume 13 of IAS/Park City Mathematics Series. AMS and IAS/Park City Mathematics Institute, 2007.

[14] H. S. Mortveit and C. M. Reidys. Discrete, sequential dynamical systems. Discrete Math., 226:281-295, 2001.

[15] I. Novik, A. Postnikov, and B. Sturmfels. Syzygies of oriented matroids. Duke Math. J., 111:287-317, 2002.

[16] O. Pretzel. On reorienting graphs by pushing down maximal vertices. Order, 3(2):135-153, 1986.

[17] J.-Y. Shi. The enumeration of Coxeter elements. J. Algebraic Combin., 6:161-171, 1997.

[18] J.-Y. Shi. Conjugacy relation on Coxeter elements. Adv. Math., 161:1-19, 2001.

[19] D. E. Speyer. Powers of Coxeter elements in infinite groups are reduced. Proc. Amer. Math. Soc., 137:1295-1302, 2009.

[20] J. R. Stembridge. On the fully commutative elements of Coxeter groups. J. Algebraic Combin., 5:353-385, 1996. 